\title{
Newsfronts
}

\section{Immaculate Conception for Stem Cells}

Researchers have successfully derived pluripotent stem cells from macaque oocytes, and their technique could provide researchers and clinicians with a promising alternative to stem cells obtained directly from dissociated embryos.

Stem cell research remains one of the few areas of medical study that one might just as easily read about in a political position paper, religious tract, or legal journal as in a conventional scientific publication. At the center of the raging controversy is the source from which pluripotent stem cells are, according to many researchers, ideally derived: viable human embryos. Although stem cells can be obtained from some adult tissues, such as the bone marrow, these are generally believed to be considerably less versatile than embryonically derived cells.

Parthenogenesis, the activation and subsequent development of an unfertilized oocyte, offers an alternative possibility for obtaining fully functional stem cells. In nature, this process occurs in a wide variety of species but does not exist in placental mammals. Such activation has been achieved experimentally, however, and oocytes activated in vitro (parthenotes) can successfully survive and develop for a number of days or even weeks after transplantation into the womb of a surrogate mother. These growing parthenotes consist entirely of embryonic tissue and are apparently incapable of reaching full maturation.

In an effort to examine this potential alternative to embryonic stem cells, Kent Vrana at the Wake Forest University School of Medicine (Winston-Salem, NC) and his colleagues isolated oocytes from three different cynomolgus monkeys, and chemically treated them to induce activation, resulting in the production of a number of blastocysts. These in turn were dissociated and cultured on feeder cells, leading to the generation of the stable stem cell line Cyno-1 (Proc. Natl. Acad. Sci. USA, 30 September). These cells were karyotypically normal and showed many phenotypic markers typical of embryonic stem (ES) cells-even after two years of maintenance and propagation.

Also like ES cells, the Cyno-1 line proved capable of differentiation into a wide variety of specialized cell types. Several weeks after injection into the peritoneal cavity of SCID mice, the Cyno-1 cells had developed into teratomas containing terminally differentiated skin, cartilage, bone, and muscle cells, among others. Cyno- 1 cells could also be cultured under conditions that led them to differentiate into functional dopaminergic neurons, suggesting that these cells could provide potential therapeutic solutions for a variety of neurodegenerative disorders.

Vrana and his colleagues are still in the process of investigating the impact of the Cyno-1 line's uniparental lineage and have not yet determined whether these cells are under any limitations relative to conventional, biparentally derived ES cells. However, their new findings suggest the possibility of a powerful new tool that may allow researchers to conduct stem cell research without quite so much controversy.

\section{— Michael Eisenstein}

\section{A Protein in Fat That Helps Keep You Thin?}

A protein expressed in a specific subset of fat cells may provide new insights into the regulation of energy balance and fat metabolism.

Contrary to general opinion, there is such a thing as 'good' fat. When most people think of fat, they're thinking of white adipose tissue (WAT), which serves to store 'fuel' for the excess energy needed during the course of metabolic processes; when rising energy stores are underused, the WAT deposits grow.

Brown adipose tissue (BAT), on the other hand, is primarily involved in thermogenesis, and its activity is essential for the regulation of body temperature in various small mammals and in animals that hibernate. BAT achieves this through the action of an 'uncoupling' protein (Ucp1) present in the membranes of the many mitochondria found in the cells of this tissue. The normal metabolic process produces heat as a by-product, but within BAT, Ucp1 short-circuits the process and makes the generation of heat the primary result of metabolism. Thus, BAT participates in both the modulation of ATP synthesis and the regulation of body heat.

Defects in BAT have also been linked to obesity, and mice lacking brown adipocytes suffer from both obesity and hyperglycemia with insulin resistance. However, mice lacking Ucp1 are of normal weight, suggesting that other BAT-specific genes may be involved in the regulation of body weight. Peng Li and her colleagues at the Institute of Molecular and Cell Biology (Singapore) decided to investigate the potential role of Cidea, a mitochondrial protein highly expressed in BAT (but not in WAT), in this regulatory function; to this end, they generated a knockout mouse strain lacking Cidea expression (Nat. Genet., September).

Upon gross examination, the mice lacking the Cidea protein seemed normal, healthy, and fertile. However, closer inspection revealed that these mice had a substantially elevated core body temperature, and increased metabolic function, as indicated by a $10 \%$ increase in oxygen consumption in comparison with wild type animals. Though roughly the same size as a normal mouse, mice lacking Cidea showed a substantial decrease in WAT and a $62 \%$ reduction in adiposity. The knockout mice also seemed capable of metabolizing triglycerides at an increased rate and showed relatively reduced blood glucose levels after consumption of a high-fat diet or direct injection of glucose.

Li's group identified a direct interaction between Cidea and Ucp1 and, in a series of co-expression assays in yeast, found evidence suggesting that Cidea can directly inhibit the uncoupling process. Deregulation of Ucp1 could explain the lean phenotype observed in the mice lacking Cidea, and may indicate an important role for Cidea in the management of energy regulation and adiposity.

- M.E. 